\title{
Scattering of Light by Hydrosol Particles Suspended in Coastal Waters
}

\author{
Eugeny B. Shybanov *, and Vladimir I. Haltrin ${ }^{\dagger}$ \\ * Marine Hydrophysical Institute of the Ukrainian Academy of Sciences, Optics Department, \\ 2 Kapitanskaya St., Sevastopol, 99011, Crimea, Ukraine, e-mail: <lee@alpha.mhi.iuf.net>. \\ † Naval Research Laboratory, Ocean Optics Section, Code 7333 Stennis Space Center, MS 39529-5004, USA. \\ Phone: (228) 688-4528, e-mail: <haltrin@nrlssc.navy.mil>, web page: <http://www7333.nrlssc.navy.mil/ haltrin>
}

\begin{abstract}
A new and efficient computational approach to calculate Mie scattering of light by spherical particles, including very large ones, is presented. The code based on this approach is capable to compute light scattering by absorbing particles with size parameters up to sixteen million, and by non-absorbing spherical particles with size parameters as large as one billion. This feature is very important for modeling scattering properties of coastal ocean waters containing suspended sand grains, clay and large detritus particles.
\end{abstract}

\section{INTRODUCTION}

Presented below approach allow us to compute Mie light scattering properties of very large particles like grains of sand, detritus and clay. These computations are very important for optics of coastal waters contaminated with terrigenic particles and dead organic matter. Results and analysis of these computations allow us to extend existing models of seawater optical properties [1-3] to include components that are specific to coastal environment. The preliminary results of using programs based on this approach to calculate optical properties of sand particles are very promising [4-6]. The approach is also applicable to smaller particles with results identical to the results produced by existing algorithms.

The theory of light interaction with scattering and absorbing medium includes two types of problems: direct and inverse. The direct problem deals with scattering of light in the medium provided that the shape, size, internal structure, composition, and dielectric permeability of particles, and properties of radiation, such as wavelength, intensity and degree of polarization, are known. In the classical case of independent scatterers such problem is rigorously formulated and reduces to the mathematical calculations. In many practical cases we are interested in inverse problem to determine properties of medium through characteristics of scattered light. Quite often the only information we have is an intensity of light scattered in one direction or, less often, in several ones. To correctly solve the inverse problem we should know vector amplitude and phase of the light field scattered in all directions together with the field inside particle. It is clear that without knowledge of additional information it is impossible to solve the inverse problem. One of the ways to simplify this problem consists of reducing the number and variability range of medium parameters with consecutive election of major factors that represent main features of light scattering and absorption. The simplification of inverse problem may be obtained through analysis of the results of the direct problem for particular physical composition of scattering and absorbing medium.

The most important and exactly solvable problem in the theory of scattering and absorption of light by scattering particles is a problem of light scattering by a sphere with arbitrary radius and refractive index. While the mathematical solution to the problem of light scattering on a homogeneous sphere is solved about century ago [7] the practical implementation of this solution became possible only with development of powerful computers. In spite of the fact that scattering particles in atmosphere and ocean are non-spherical and non-homogeneous, results of Mie theory [7], averaged over particles size distribution, allow us in the first approximation to describe optical effects from scattering by randomly oriented polydisperse non-spherical particles. The Mie theory also describes multitudes of effects related to scattering, effects that are very difficult to understand using only qualitative approach.

Mathematical rigorousness of the solution of light scattered by a spherical particle implies that the numerical scattering algorithm used to compute this scattering should be precise and stable. Stability of the basic procedures of the algorithm allows to improve computational effectiveness of more difficult problems such as scattering by a coated particle. In many cases the speed of calculations is also important. Concerning the speed of execution and stability at large size parameters the programs, that are based on a proposed algorithm, have no known prototypes in the world. The increase in speed of computations achieved due to improvement of the 
OCEANS 2002 MTS-IEEE Proceedings, vol.4, IEEE Catalog Number: 02CH37362C, ISBN: 0-7803-7535-1

algorithm is about 6 times. Additional performance characteristics of the algorithm are also exceed characteristics of widely known programs, such as Wiscombe's [8, 9] program. For example, the number of scattering angles can reach 3601 and a size parameter can be as large as one billion for nonabsorbing particles, and, respectively, 1801 and ten million for absorbing particles.

Below we present a short overview of Mie theory $[7,10]$ that is necessary for further references and clarity of proposed computational algorithm.

\section{DETAILS OF MIE SCATTERING COMPUTATIONS}

Let us consider a plane monochromatic light wave with a wavelength $\lambda$ and wave number $k=2 \pi / \lambda$ that falls on a spherical particle with radius $a$. The complex amplitudes of falling and scattered fields are connected with the following relationship,

$$
\left(\begin{array}{c}
E_{\perp s} \\
E_{\| s}
\end{array}\right)=\frac{e^{i \cdot k \cdot r}}{-i \cdot k \cdot r}\left(\begin{array}{cc}
S_{1} & 0 \\
0 & S_{2}
\end{array}\right)\left(\begin{array}{c}
E_{\perp} \\
E_{\|}
\end{array}\right) .
$$

Coefficients of scattering matrix are expressed in the form of the following infinite sums [10]:

$$
\begin{aligned}
& S_{1}=\sum_{n=1}^{\infty} \frac{2 n+1}{n(n+1)}\left(a_{n} \pi_{n}+b_{n} \tau_{n}\right), \\
& S_{2}=\sum_{n=1}^{\infty} \frac{2 n+1}{n(n+1)}\left(a_{n} \tau_{n}+b_{n} \pi_{n}\right),
\end{aligned}
$$

here $\pi_{n}$ and $\mathrm{t} \tau_{n}$ are functions of scattering angle $\theta$; and coefficients $a_{n}, b_{n}$ depend on dimensionless size parameter $x=k a$. Expressions (2) represent decomposition of a scattered wave on multipole components. To obtain energetic scattering characteristics we need to compute Mueller $4 \times 4$ matrix $S_{i k}$ with the following elements:

$$
\begin{gathered}
S_{11}=\frac{1}{2} \operatorname{Re}\left(S_{1}^{2}+S_{2}^{2}\right), S_{12}=\frac{1}{2} \operatorname{Re}\left(S_{2}^{2}-S_{1}^{2}\right), \\
S_{33}=\frac{1}{2} \operatorname{Re}\left(S_{2}^{*} S_{1}+S_{2} S_{1}^{*}\right), \\
S_{34}=-\frac{1}{2} \operatorname{Im}\left(S_{2}^{*} S_{1}-S_{2} S_{1}^{*}\right) .
\end{gathered}
$$

The physical meaning of a size parameter $x$ is a number of wavelengths that fit into a perimeter of a particle with radius $a$. It means that input from a multipole of $n^{\text {th }}$ order at $n>x$ is diminishing quickly with the increase of $n$ According to Wiscombe [8], the maximum number of important elements $n_{\max }$ in the infinite series (2) may be estimated according to the regression:

$$
n_{\max }=x+L x^{1 / 3}+2,
$$

here the coefficient $L$ varies between 4 and 6 depending on the value of $x$. The angular functions $\pi_{n}, \quad \tau_{n}$ are expressed through the Legendre polynomials of the first kind:

$$
\pi_{n}=\frac{P_{n}^{1}}{\sin \theta}, \tau_{n}=\frac{d P_{n}^{1}}{d \theta} .
$$

These functions are computed using following recurrent relationships:

$$
\begin{gathered}
\pi_{n}=\frac{2 n-1}{n-1} \mu \pi_{n-1}-\frac{n}{n-1} \pi_{n-2}, \\
\tau_{n}=n \mu \pi_{n}-(n+1) \pi_{n-1},
\end{gathered}
$$

here $\mu=\cos \theta, \pi_{0}=0, \pi_{1}=1$. The coefficients $a_{n}$, $b_{n}$ are expressed through Bessel-Ricatti functions and their derivatives:

$$
\begin{aligned}
& a_{n}=\frac{m \psi_{n}(m x) \cdot \psi_{n}^{\odot}(x)-\psi_{n}(x) \psi_{n}^{\odot}(m x)}{m \psi_{n}(m x) \cdot \xi_{n}^{\odot}(x)-\xi_{n}(x) \cdot \psi_{n}^{\odot}(m x)}, \\
& b_{n}=\frac{\psi_{n}(m x) \psi_{n}^{\odot}(x)-m \cdot \psi_{n}(x) \psi_{n}^{\odot}(m x)}{\psi_{n}(m x) \xi_{n}^{\odot}(x)-m \cdot \xi_{n}(x) \psi_{n}^{\odot}(m x)},
\end{aligned}
$$

here $m$ is a complex refraction index of a scattering sphere relative to the refraction index of surrounding medium, and the wavelength in the expression for $x$ includes wavelength of the light in surrounding medium. The Bessel-Ricatti functions, both for complex and real arguments, satisfy the following recurrence relationship:

$$
z_{n+1}(x)=\frac{2 n+1}{x} z_{n}(x)-z_{n-1}(x)
$$

with the following expressions for initial terms of these functions:

$$
\begin{gathered}
\psi_{0}(x)=\sin x, \quad \psi_{1}(x)=\frac{\sin x}{x}-\cos x, \\
\xi_{n}(x)=\psi_{n}(x)-i \chi_{n}(x),
\end{gathered}
$$


OCEANS 2002 MTS-IEEE Proceedings, vol.4, IEEE Catalog Number: 02CH37362C, ISBN: 0-7803-7535-1

$$
\chi_{0}(x)=\cos x, \quad \chi_{1}(x)=\frac{\cos x}{x}+\sin x .
$$

To simplify computation of coefficients $a_{n}$ and $b_{n}$ Aden [10] introduced the following logarithmic derivative:

$$
D_{n}(x)=\frac{d}{d x} \ln \psi_{n}(x) \equiv \frac{1}{\psi_{n}(x)} \frac{d \psi_{n}(x)}{d x} .
$$

Using (10) and the following recursions for the Bessel-Ricatti functions,

$$
z_{n}^{\prime}(x)=z_{n-1}(x)-\frac{n z_{n}(x)}{x}
$$

we can rewrite Eqs. (7) as follows:

$$
\begin{aligned}
& a_{n}=\frac{\left[D_{n}(m x) / m+n / x\right] \psi_{n}(x)-\psi_{n-1}(x)}{\left[D_{n}(m x) / m+n / x\right] \xi_{n}(x)-\xi_{n-1}(x)}, \\
& b_{n}=\frac{\left[m D_{n}(m x)+n / x\right] \psi_{n}(x)-\psi_{n-1}(x)}{\left[m D_{n}(m x)+n / x\right] \xi_{n}(x)-\xi_{n-1}(x)},
\end{aligned}
$$

with the logarithmic derivative (10) satisfying the recursion relationship:

$$
D_{n-1}(x)=\frac{n}{x}-\frac{1}{D_{n}(x)+n / x} .
$$

Relationships (12) are more convenient for numerical computations than Eqs. (7) because they include only one parameter, a logarithmic derivative $D_{n}$, which is a function of complex argument. The Bessel-Ricatti functions in (12) are functions of real argument $x$. Another computational advantage of (12) over (7) is in the recursion relationship (13) that employs only one previous value of function instead of two values used in (8).

Computational problem of recurrence relationships at $n \rightarrow \infty$ is coupled with different types of convergencies for functions $D_{n}, \chi_{n}, \psi_{n}$. Numerical representation of these functions has limited precision. As a consequence, for any arbitrary values of Bessel-Ricatti functions we have some errors $\varepsilon_{n-1}$, and $\varepsilon_{n}$. These errors can be represented as linear combinations of Bessel-Ricatti functions:

$$
\varepsilon_{k}=C_{1} \psi_{k}+C_{2} \chi_{k}, \quad k=n-1, n .
$$

It is clear from (14) that, if at any particular computational step we achieve an ideal precision, we would not eliminate the error of computation, because it had been carried out from previous steps. The relative error for, let say, function $\psi$ is:

$$
\delta \psi_{k}=C_{2} \chi_{k} / \psi_{k}
$$

Starting from a certain values of $k$ the function $\chi$ gradually grows, while the function $\psi$ diminishes. As a result, in the range of continuous increase of $\chi$ both upward recursions for $\psi$ and $D$ diverge. Similarly, computation of function $\chi_{n}$ with a downward recursion also diverges. A minimum number of iterations $\Delta n$ can be estimated as

$$
\Delta n=L \cdot x^{1 / 3}+2
$$

here $L$ is a constant with a value in the range from 7 to 8. Similar estimates of logarithmic derivative give necessary iterations number and a range of $n$ 's where the downward recurrence converges:

$$
n>m x, \quad \Delta n=L(m x)^{1 / 3}+2 .
$$

This estimate is evident if the refractive index $m$ is real. If $n_{0}$ is a limiting number in a series given by (4), then the initial values of $n$ in downward recursion procedures are less than

$$
\begin{gathered}
n_{0 \psi}=n_{0}+L x^{1 / 3}+2, \\
n_{0 D}=n_{0}+(m-1) x+L(m x)^{1 / 3}+2 .
\end{gathered}
$$

In a majority of algorithms that compute coefficients $a_{n}, b_{n}$ it is typical to calculate $\psi_{n}$ using an upward recursion. The function $\psi_{n}$ diverges when $n>x$. As a consequence, the computer rounding error influences the value of $L$ in (4). With the increase of size parameter $x$ the rounding errors accumulate. This causes the optimal value of $L$ to be dependent on $x$. As a consequence, smaller values of $L$ become preferable.

This problem is not as important for computations of extinction and scattering efficiencies $Q_{e}$ and $Q_{s}$. These values are determined by the following equations:

$$
\begin{aligned}
& Q_{e}=\frac{2}{x^{2}} \sum_{n=1}^{\infty}(2 n+1)\left(\left|a_{n}\right|^{2}+\left|b_{n}\right|^{2}\right), \\
& Q_{s}=\frac{2}{x^{2}} \sum_{n=1}^{\infty}(2 n+1) \operatorname{Re}\left(a_{n}+b_{n}\right),
\end{aligned}
$$


OCEANS 2002 MTS-IEEE Proceedings, vol.4, IEEE Catalog Number: 02CH37362C, ISBN: 0-7803-7535-1

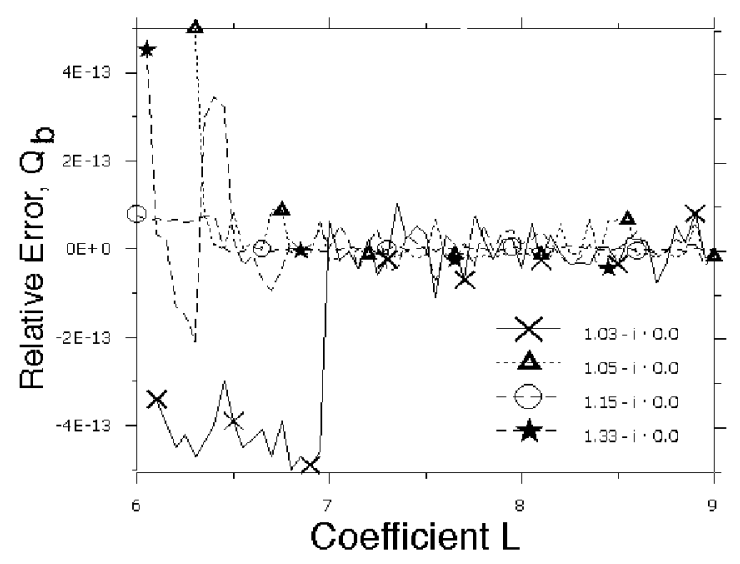

Fig. 1. Relative error of computing scattering to $180^{\circ}$ for non-absorbing particles with various refraction indices and size parameter $x=2 \cdot 10^{4}$ as a function of coefficient $L$ in equation (4).

and they converge rapidly. As our computational examples show, all 16 decimal digits coincide at $L>3.7$. The asymmetry parameter,

$$
\begin{array}{r}
Q_{g}=\frac{4}{x^{2}} \sum_{n=1}^{\infty}\left(\frac { n ( n + 2 ) } { n + 1 } \operatorname { R e } \left(a_{n} \cdot a_{n+1}^{*}+\right.\right. \\
\left.\left.b_{n} b_{n+1}^{*}\right)+\frac{2 n+1}{n(n+1)} \operatorname{Re}\left(a_{n} b_{n}^{*}\right)\right),
\end{array}
$$

also converges quickly.

Another problem exists with the calculation of an efficiency factor at 180 degrees, or a radar backward cross-section:

$$
Q_{b}=\frac{1}{x^{2}}\left[\sum_{n=1}^{\infty}(-1)^{n}(2 n+1)\left(a_{n}-b_{n}\right)\right]^{2} .
$$

The radar backward cross-section may be used as a test value for precision of phase function calculations in the whole range of angles between 0 and 180 degrees. Computational errors of $Q_{b}$ as a function of parameter $L$ are shown in Fig. 1. The computations are made for size parameter $x=20000$ with $\psi_{n}$ computed by downward recursion. Fig. 1 shows that $Q_{b}$ could not be computed with the ideal precision, and minimal errors in back-scattering calculations correspond to $L>7$. This range is not acceptable for computations of $\psi_{n}$ by upward recursion. In this case, if we accept $L=4$, the relative errors of $Q_{b}$ for analyzed refraction indices will be in the range of

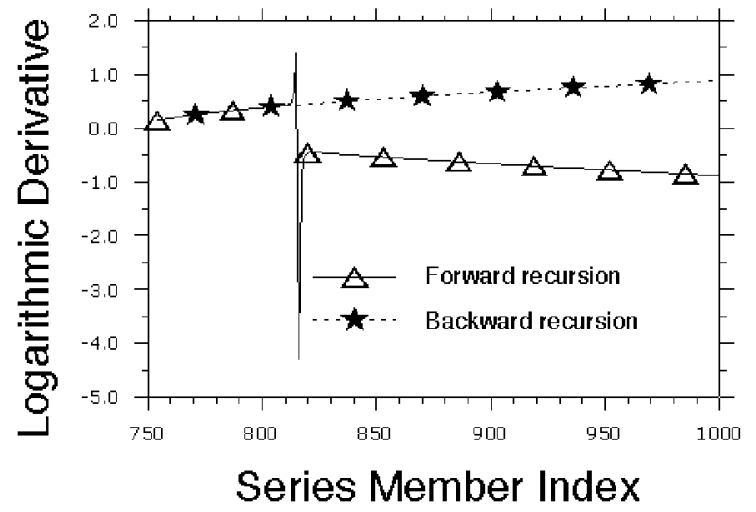

Fig. 2. Comparison of forward and backward recursion methods in computing logarithmic derivative for the case of air bubble with size parameter $x=1000$ in water.

$7 \cdot 10^{-8}$. If this level of errors is acceptable, then according to Eqs. (18), for non-absorbing particles with $m>1$ computations of Bessel functions and their derivatives could be accomplished by upward recursion method. At the same time, both methods of upward and downward recursion do not produce identical results. For example, for the refractive index $m=1.5+i \cdot 0$ a comparison of upward and downward recursion results shows that at $x \sim 10^{5}$ corresponding values of the real part of $D_{1}(m, x)$ coincide with 10 -digit precision, while at $x \sim 10^{6}$ these values coincide with 9-digit precision.

This difference is due to accumulation of error in logarithmic derivative computation by the downward recursion method. Similar error accumulation is also specific for the direct recursion method. Consequently, provided that the upward recursion converges, the difference between upward and downward recursions consists in the fact that in the case of upward recursion the lower terms $a_{n}, b_{n}$ are computed with higher precision, while in the case of downward recursion the higher terms are computed with higher precision.

Certain interest represent non-absorbing particles with refractive index less than one (like air bubbles in water). When the upward recursion of logarithmic derivative should start to diverge at $n>m x$, the formal results of computations look credible. For example, for air bubbles in water $(m=0.75)$ at $x=1000$, values of efficiency factors for scattering and extinction coincide and are equal to $Q_{s}=Q_{e}=2.0018805$. At the same time the Wiscombe's program [8] gives values of $Q_{s}=Q_{e}=1.9979082$. To analyze this result in Fig. 2 we show results of computations by upward recursion. 
OCEANS 2002 MTS-IEEE Proceedings, vol.4, IEEE Catalog Number: 02CH37362C, ISBN: 0-7803-7535-1

It is necessary to note that in upward recursion algorithm we used 10-byte representation of floating numbers. In spite of this the recursion completely diverges at value of $n \approx 815$. The identity of recurrent relationships for functions $\chi$ and $\psi$ causes at iteration numbers $n>815$ to compute a logarithmic derivative of $\chi$. Based on the value of $n$ at divergence point $(n \approx 815)$ the estimate for $L$ in Eqs. (16)-(18) is: $L \approx 7.15$.

Computational specifics of scattering by absorbing particles have been analyzed earlier by Dave [12]. In his paper Dave showed that the upward recursion of logarithmic derivative for the real part of refractive index $\operatorname{Re}(m)=1.342$ diverges. As a solution he proposed to use the procedure of downward recursion starting from

$$
n_{0 D}=1.1|m \cdot x|+1 \text {. }
$$

As an initial value for downward recursion of logarithmic derivative Bohren and Huffman [13] use the value of

$$
n_{0 D}=\max \left(n_{\max },|m x|\right)+15,
$$

where $n_{\max }$ is found from (8) at $L=4$. As a convergence criterion for an upward recursion Bohren and Huffman [13] proposed an non-equally criterion, $|\operatorname{Im}(m x)|<80$. According to a discussion above, such criterion should also depends on a real part of refractive index. The divergence of upward recursion at $\operatorname{Re}(m)>1$ and $\operatorname{Im}(m) \neq 0$ means that the downward recursion should converge at values of $n$ significantly smaller than $\operatorname{Re}(m x)$ or, at largest values of $x$, at $n_{0 D}$ determined by (18).

Lentz [14] proposed a specific algorithm to evaluate initial values of logarithmic derivative $D_{n \text { max }}$. This algorithm became a basis for a wellknown and stable Wiscombe's program [9] that is capable to compute Mie scattering values for size parameters $x$ up to 20,000 . The Lentz's method is effective for absorbing particles and $\operatorname{Re}(m)<1$, otherwise the number of iterations of the method is about $(m-1) x$. For weakly absorbing particles more elaborate initialization method of Lentz is less effective than the direct method of logarithmic derivative calculations that uses (13) starting from $n_{0 D}$, determined by Eqs. (18) or (23). Another shortcoming of the Lentz's method is a requirement to check division by a small number. To eliminate uncertainty of zero by zero division Cachorro and Salcedo [15] proposed an improved method of logarithmic derivative initialization. By analyzing various sources of computational errors in Mie calculations Cachorro and Salcedo came to a conclusion that it is possible to make such computations for any arbitrary values of refraction index and size parameter.

\section{ALGORITHM}

By analyzing major features of logarithmic derivative convergence we propose a new improved method for computation of logarithmic derivative. The purpose of the improvement is to increase speed, precision and stability of Mie computations.

In the case of natural scattering particles their size distribution function may be regarded as continuous. This means that the procedure to compute optical properties of these particles consists in integration over size distribution. The accuracy of final results depends on the number of integration points. To choose the proper integration method it is necessary to understand what functions may be integrated. Functional dependence of scattering properties on size parameter is not smooth. According to 16, 17] resonance structures are revealed for both extinction and absorption. For this reason the integration technique using Simpson's or, even worse, quadrature formula leads to a significant increase in error.

For the optimal choice of integration grid, in respect to the integration precision, we should possibly pre-compute a sequence of integral parameters $Q_{i}=\left(Q_{e}, Q_{s}, Q_{a s}, Q_{b}\right)$ at different number of grid points $k=k_{0}, k_{0}+1, \ldots, k_{0}+n$ and then to choose a proper value of $k$. A possible criterion of optimal greed is a minimum of distance to an average point:

$$
\varepsilon_{k}=\sum_{j} \frac{\left(Q_{j}^{k}-\overline{Q_{j}}\right)^{2}}{\sigma_{j}+\sigma_{0}}
$$

here $\sigma_{j}$ is a dispersion of $j^{\text {th }}$ efficiency factor, and $\sigma_{0}$ is an acceptable value of dispersion.

The criterion to use this procedure in Mie calculations is determined by the effectiveness of $a_{n}$, $b_{n}$ computations, and, consequently, by the effectiveness of logarithmic derivative computation. As our testing shows, the duration of one iteration using Lentz's method and Wiscombe's procedure on a Celeron-333 CPU is about $1.5 \cdot 10^{-5}$ seconds. As a comparison, our direct coding of (13) using high-level computing language allows us to increase 20 times the computational speed of one recursion. The same procedure implemented by one of the authors (EBS) 
OCEANS 2002 MTS-IEEE Proceedings, vol.4, IEEE Catalog Number: 02CH37362C, ISBN: 0-7803-7535-1

as an assembler code executes 60 times faster than the Wiscombe's procedure [9]. It is fair to mention that the coded version of Lentz's method is not optimal in respect to complex arithmetic. At the same time such large difference in execution speed doubts effectiveness of numerical algorithms to investigate convergence of logarithmic derivative inside the Mie code. Concerning the approach applied to large absorbing particles to compute iterations starting with $n_{0 D}$ determined by (18), we came to the conclusion that this limit is largely overestimated.

The essence of proposed method is to restrict from the top the number of possible iterations for absorbing particles. Let us note, that because the efficiency of computing using recurrent relation (13) is very high, we have no need to find a precise mathematical value.

In the beginning, let us consider some exceptions: (a) for a non-absorbing particles let us begin our computations of logarithmic derivative and function $\psi$ using downward recursion starting with $n_{0 \psi}$ and $n_{0 D}$, defined by (18); (b) for $\operatorname{Re}(m)<1$ we can accept $n_{0 D}=n_{0 \psi}$. Alternatively, consider the case when $\operatorname{Re}(m)>1, \operatorname{Im}(m) \neq 0$. Now let us compute a value of logarithmic derivative $D_{n}^{\alpha}$ at $n \approx x+L \cdot x^{1 / 3}, L=7$, using an over-estimated value of $n_{0 D}$ taken from (18). Next, let us consecutively lower value of $n_{0 D}$ until the absolute value of the difference between computed value of $D_{n}$ and value of $D_{n}^{\alpha}$ exceeds the predetermined value of $\varepsilon$. The value $k=n_{0 D}-n$ then determines the necessary number of iterations in a more rigid sense than in the Lentz's method.

From the results of computations, displayed in Fig. 3, it is clear that for all $\operatorname{Im}(m) \neq 0$ the iteration number $k$ as a function of size parameter $x$ at $x \rightarrow \infty$ approaches certain asymptotic value. In the limit of small values of $x$ the function $k(x)$ is restricted from above by the value of iterations for non-absorbing case $k_{n \alpha}$, which is determined by (18).

Relative to the efficiency of computations, the simplicity of numerical algorithm is more important compared to its mathematical rigorousness. For this reason let us determine the number of necessary iteration as

$$
n_{i t}=\min \left(k_{n \alpha}, k_{a c}\right),
$$

where $k_{a c}$ is the asymptotic value of iteration numbers as a function of the refractive index.

To analyze the functional dependence of maximum number of iterations on complex
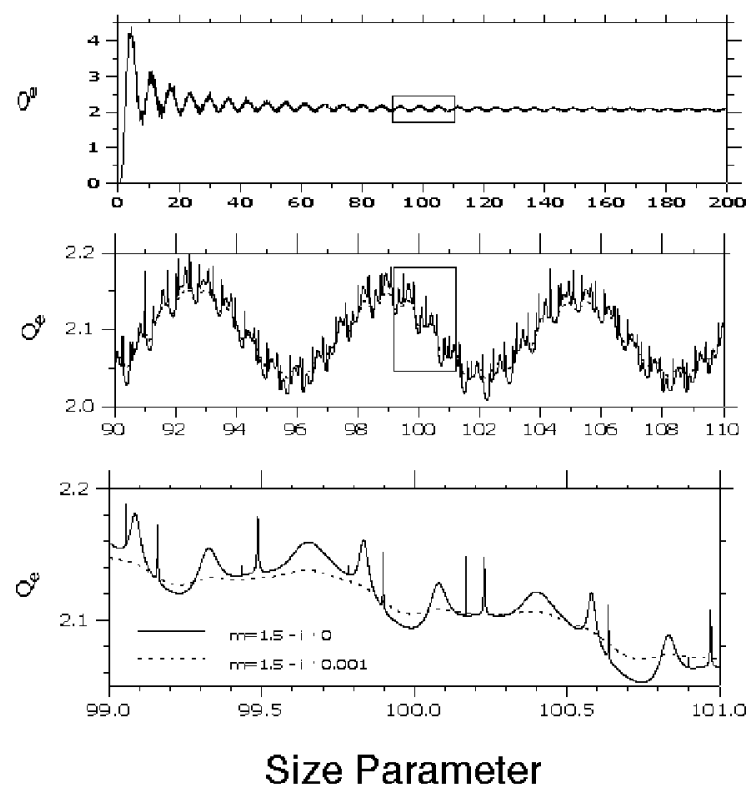

Fig. 3. Dependence of a number of iterations for computing logarithmic derivative from size parameter for various refraction indices.

refraction index, let us plot $k_{a c}$ as functions of imaginary part of refractive index. The number of iterations shown in Fig. 3 are applicable to compute logarithmic derivative using (4) with $L=7$. As it was mentioned above, for computing $\psi$ with upward recursion, $L$ should be of the order of 4 . Consequently, the number of iterations should be increased by $\Delta n \approx 3 \cdot x^{1 / 3}$.

Some results of computations for the case of refractive index in the range between 1.01 and 3 are shown in Fig. 4. In order to determine reliable value of maximum at small values of absorption the value of size parameter $x$ was continuously varied from small values to $5 \cdot 10^{7}$. The imaginary part of refractive index varied from 0.001 to 2 . At small absorption values the number of iterations given by (26) is less then $k_{a c}$ if $x$ is not very large (the program with absorption works up to $x=16000000$ ). One of the major feature of the curves displayed in Fig. 4 is a similarity of linear dependencies of the maximum number of iterations as a function of reverse imaginary part of refractive index. Linear dependence in a log-log scale implies a power law. The exponent of this power law is very close to unity and is always less than 1.009.

Consequently, we can conclude that the asymptotic number of iterations is reversely proportional to the imaginary part of refractive index. Discrepancies to this behavior are developed when absorption increases, and these differences are 
OCEANS 2002 MTS-IEEE Proceedings, vol.4, IEEE Catalog Number: 02CH37362C, ISBN: 0-7803-7535-1

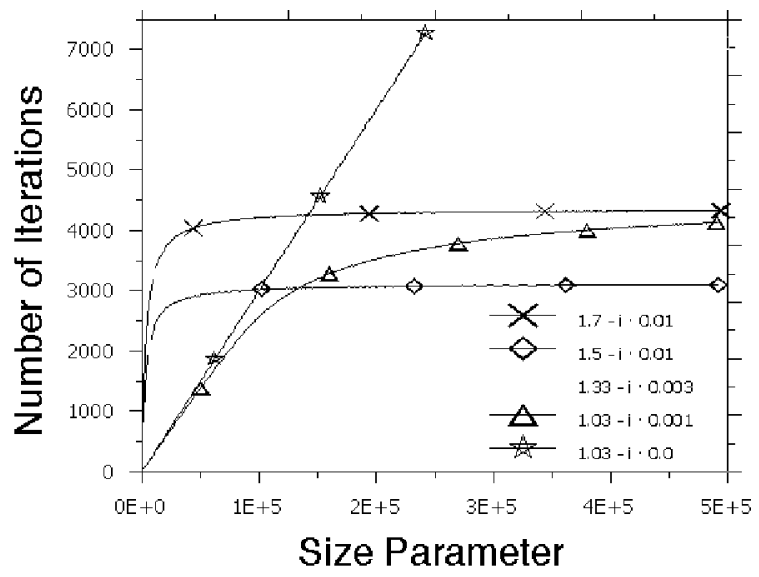

Fig. 4. Dependence of a number of iterations for computing logarithmic derivative as a function of reverse absorption coefficient.

higher for smaller values of the real part of refractive index. Studies of the behavior of the maximum iteration number with the increase of $k>\operatorname{Re}(m)$ revealed a linear type of dependence in this area. The proportionality coefficient in this dependence is independent on refraction index and is equal to 20 .

The Fig. 4 also shows that the coefficient of asymptotic dependence of $1 / k$ in logarithmic scale (shift parameter) is closely connected with the real part of refractive index. A close analysis of numerical results shows that the precise empirical relationship that describes computed dependencies shown in Fig. 4 can be written as:

$$
N_{i t}=20 k+\frac{m}{k}\left(20.3 m-5.8-\frac{2.4}{m-0.8}\right)+30 .
$$

This relationship may be used also in the area of high values of absorption. The real part of refractive index here is denoted as $m$, and the absolute value of imaginary part as $k$.

Empirical equation (27) by as far as $60 \%$ overestimates a number of necessary iterations in the range of strong absorption. Nevertheless, the average overestimation over the whole set of computational values is only in the range of $2 \%$.

Comparison of computational results of maximum necessary iterations with the values estimated using (27) is shown in Fig. 5. The figure shows that (27) with a high accuracy defines the number of necessary iterations. Equation (26) with Eqs. (18) and (27), due to the condition of exceeding a number of iterations above necessary or defined by the Lentz algorithm, systematically exceeds the necessary value. Maximum relative overestimation of a number of iterations for the curves shown in Fig. 3

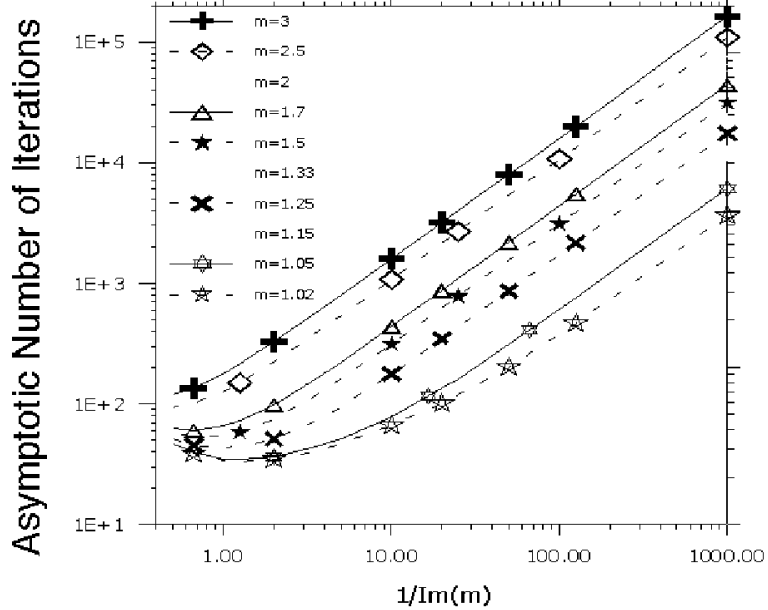

Fig. 5. Upper estimate of a maximum number of iterations given by (27) compared with the values obtained from a numerical experiment.

is equal to $1.46-1.57$ for $m>1.03$ and approximately 2.12 for $m=1.03$. Visible discrepancy for optically soft particles is due to a less rigid estimation of the number of iterations for the case without absorption. In this case to approximate results of computational experiment we can use the following equation:

$$
n_{i t}=(m-1) x+L(m x)^{1 / 3}-L x^{1 / 3}+1 .
$$

This correction, however, insignificantly changes effectiveness of computational code for the case of large particles due to the large values of size parameter $x>x^{1 / 3}$.

As our numerical testing shows, (27) is applicable to the following extreme values of optical constants: (a) $m=8.9 ; k=0.69$, the case of refractive index of water at wavelength equal to $10 \mathrm{~cm}$; (b) $m=37$, $k=41$, the case of refractive index of platinum at wavelength equal to $10 \mathrm{mkm}$.

Consequently, for all realistic values of refractive index $m>1$ the maximum iteration number may be estimated using (27). In spite of the approximate method to estimate the number of iterations of the logarithmic derivative, the execution speed of this part of the Mie calculations is about 10 times higher than the execution speed of corresponding fragment in Wiscombe's code. As a whole, the execution speed of the code determined by the effectiveness of computing summations defined by (2). The coefficients $a_{n}, b_{n}$ given by (2) does not depend on angle; and angular functions $\pi, \tau$ does not depend on optical properties and size parameter. As a consequence, these values are computed independently. 
OCEANS 2002 MTS-IEEE Proceedings, vol.4, IEEE Catalog Number: 02CH37362C, ISBN: 0-7803-7535-1

\section{COMPUTATIONS OF PHASE FUNCTION}

Among others, there are two effective methods to compute phase function:

a) To compute consecutively coefficients $a_{n}, b_{n}$ and summate them at each angle. The program based on this method and utilizing approach of this paper is written and available for public use [18]. This program can compute Mie optical parameters and phase function of scattering with size parameters $x$ up to one billion $\left(x \sim 10^{9}\right)$. The consecutive calculation of coefficients $a_{n}$ and $b_{n}$ is possible only with upward recursion. This restricts this program to calculate Mie scattering only on a weakly absorbing particles.

b) To compute all values of $a_{n}$ and $b_{n}$ and to summate complex amplitudes separately for each scattering angle. This method allows to use a downward recursive procedure.

The shortcoming of the downward procedure consists of the necessity not only to compute, but to save all series terms into a dynamic memory (RAM), which increases the requirements to the computer used for calculations.

In some special computational cases, especially of polydisperse large particles with the requirement to compute results for large number of scattering angles, it became very important to create a memory-efficient program.

In ordinary case we compute downward recursion for each term of series, i. e. about $n_{0 D}-i$ iterations, where $i$ is an index of series term. In order to avoid unnecessary computations in a regime of economic memory usage we propose to use a two-pass method of downward recursion. This method consists of the following. In the first pass we compute all terms of logarithmic derivative series and also function $\psi$. At the same time we keep in memory not all computed values, but a reduced array of 'base' values that differ in indices on a fixed value of $j$. These values are stored in order to use them later as an initial values for downward recursion in the next stage of algorithm. In this stage the 'base' values of complex function $D$ and functions $\psi_{i}$ and $\psi_{i+1}$ allow us to compute a sequence of these functions in the interval $i=[k, k+j],(k=n j+1)$. We also compute the values of $a_{i}$ and $b_{i}$ in the same interval. After computation of partial sums these values are no longer necessary and the memory they occupy may be released. This method is capable to compute Mie parameters for size parameter $x>1.6 \cdot 10^{7}$ with the small memory usage equal to $640 \mathrm{~K}$. It is necessary to note that in an assembler version of the downward recursion the execution time of the first pass is

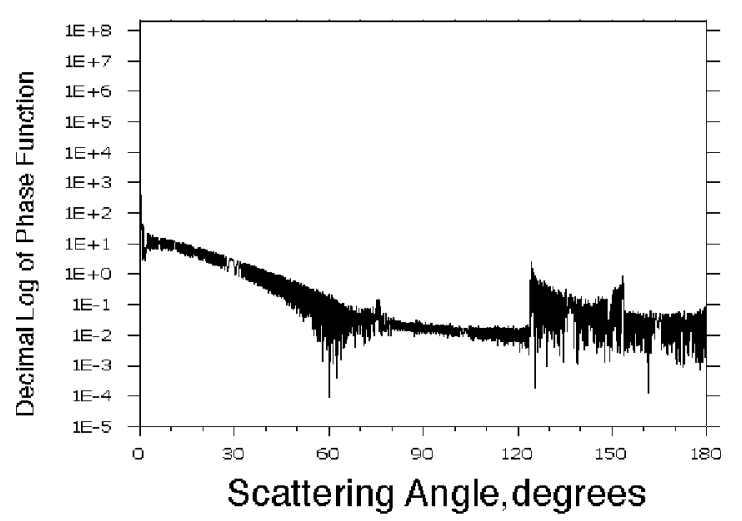

Fig. 6. Light scattering phase function by particle with relative refractive index $n=1.5$ and size parameter $x=20000$ in water.

smaller than the time necessary to compute and store functions $\psi_{i}$ and $D_{i}$ for all $i$. This effect is a result of the $j$-fold reduction in a number of CPU-RAM processes.

An important factor in an analysis of Mie scattering of light by large particles is a number of angles to compute phase function. For example, for an non-absorbing mono-disperse system of particles the angular structure of phase function becomes more and more oscillating. An example of such phase function is given in Fig. 6. The high frequency oscillations are the result of increase in the share of angular functions with large indices. Equation (4) shows that the maximum frequency of oscillations grows almost proportionally to the size parameter $x$. At the same time a polydisperse system of particles usually do not display such structure. It means that in order to produce a smooth polydisperse phase function we need to integrate using a very large number of integration points. The alternative method consists of smoothing over the aperture of receiving device. This method, as it is clear from Fig. 6 , also needs a large number of angles. All this demonstrate that the increase in speed of calculation eventually results in an improving quality of the final results.

In order to enhance effectiveness of phase function calculations let us consider the properties of functions $\pi$ and $\tau$. These functions have the following symmetry properties:

$$
\pi_{n}(-\mu)=(-1)^{n-1} \pi_{n}(\mu), \tau_{n}(-\mu)=(-1)^{n} \tau_{n}(\mu) .
$$

The series $\pi_{1}, \tau_{1}, \pi_{2}, \tau_{2,}, \pi_{n}, \tau_{n}$ are series of even and odd functions. This property of the functions $\pi$ and 
OCEANS 2002 MTS-IEEE Proceedings, vol.4, IEEE Catalog Number: 02CH37362C, ISBN: 0-7803-7535-1

$\tau$. may reduce the number of calculations. In the case when the angular grid is symmetrical relative to $90^{\circ}$, the number of calculations of functions $\pi$ and $\tau$. may be reduced twice. By computing a sum of complex intensities for even $S_{E}$ and odd $S_{O}$ angular functions, we obtain,

$$
S_{j}(\mu)=S_{E}+S_{O}, \quad S_{j}(-\mu)=S_{E}-S_{O}
$$

Another property of the angular functions, the independence on size parameter, may be exploited during an integration over size distribution. If the available dynamic memory is large enough, the values of angular functions may be computed only ones. For a large number of angular values this method can give a $60 \%$ increase in speed.

\section{CONCLUSION}

A new efficient computational algorithm to calculate Mie scattering of light by spherical particles, including very large ones, is presented. The codes are capable to compute light scattering by absorbing particles with size parameters up to sixteen million, and by non-absorbing spherical particles with size parameters as large as one billion. This feature is very important for modeling scattering properties of coastal ocean waters that contain suspended sand grains, clay and large detritus particles. The codes of Mie scattering based on the presented algorithm are available for public use [18].

\section{ACKNOWLEDGMENTS}

The author from the Naval Research Laboratory (NRL) thanks continuing support at through the VSF 73-6641-02-5 program. This article represents a NRL contribution PP/7330-02-54.

\section{REFERENCES}

[1] V. I. Haltrin, "Light scattering coefficient of seawater for arbitrary concentrations of hydrosols," J. Optical Soc. America, 16, 17151723, (1999).

[2] V. I. Haltrin, "Chlorophyll-based model of seawater optical properties," Appl. Optics, 38, 6826-6832 (1999).

[3] V. I. Haltrin,"Empirical algorithms to restore a complete set of inherent optical properties of seawater using any two of these properties," Canadian Journal of Remote Sensing, 26, 440445, (2000).
[4] V. I. Haltrin, and E. B. Shybanov "Light scattering properties of quartz particles in seawater," - in Proceedings of the International Geoscience and Remote Sensing Symposium IGARSS 2000, ed. Tammy I. Stein, IEEE, Piscataway, NJ, USA, pp. 1842-1844, (2000).

[5] V. I. Haltrin, E. B. Shybanov, R. H. Stavn, and A. D. Weidemann, "Light scattering coefficient by quartz particles suspended in seawater," in Proceeding of the International Geoscience and Remote Sensing Symposium IGARSS'99, Hamburg, Germany, ed. Tammy I. Stein, IEEE, Piscataway, NJ, USA, pp.1420-1422, (1999).

[6] V. I. Haltrin, and E. B. Shybanov, "Light scattering properties of sand particles suspended in seawater,"Oceanography, 14, 25-25 (2001).

[7] G. Mie, "Beitrage zur Optik Trüber Medien, Speziell Kolloidaler Metallösungen," Annalen der Physik, 25, 25-445, (1908).

[8] W. J. Wiscombe Mie scattering calculations: advances in technique and fast, vector-speed computer codes, NCAR/TN-140+STR, (National Center for Atmospheric Research, Boulder, Colo, USA, 1979).

[9] W. J. Wiscombe "Improved Mie Scattering Algorithm," Appl. Opt., 19, 1505-1509, (1980).

[10] H. C. Van de Hulst, Light Scattering by Small Particles, pp. 470, (Dover Publications, Inc., New York, NY, 1981)

[11] A. L. Aden, "Electromagnetic scattering from spheres with sizes comparable to the wavelength," J. Appl. Phys., 22, 1242-1246, (1951).

[12] J. V. Dave, "Scattering of electromagnetic radiation by a large, absorbing sphere," IBM J. Res. Develop., May 1, 302-313, (1969).

[13] C. F. Bohren, and D. R. Huffman, Absorption and Scattering of Light by Small Particles, (Wiley Interscience, New York, N.Y., 1983).

[14] W. J. Lentz, "Generating Bessel functions in Mie scattering calculations using continued fractions," Appl. Opt. 15, 668-671, (1976)

[15] V. E. Cachorro, and L. L. Salcedo, "New Impruvements for Mie Scattering Calculations," $J$. of Electromag. Waves and Applications, $\mathbf{5}$, 913-926, (1991).

[16] P. Chylek P., J. T. Kiehl, M.K.W. Ko "Narrow resonance structure in the Mie scattering characteristics. Appl. Optics, 17, 3019-3021, (1978).

[17] H. S. Bennett, and G. J. Rosasco "Resonances in efficiency factor for absorbtion: Mie scattering theory," Appl. Optics, 17, 491-493, (1978)

[18] The codes based on presented algorithm are available at $<$ http://www7333.nrlssc.navy.mil/ $\sim$ haltrin $>$. 